\title{
Effective presentation of immunogenicity risk assessments and related data in regulatory dossiers
}

\author{
Paul Chamberlain*,1 \\ ${ }^{1}$ NDA Advisory Services, Ltd, Grove House, Guildford Road, Leatherhead, Surrey KT22 9DF, UK \\ *Author for correspondence: Tel.: +33 5629634 37; paul.chamberlain@ndareg.com
}

The purpose of this article is to provide practical advice about how to present immunogenicity-related information in regulatory dossiers, with a particular focus on a model for an Integrated Summary of Immunogenicity to be submitted in the marketing authorization application for novel biopharmaceutical products in ICH regions (EU, USA and Japan). A format that links the analysis of potential risk factors to a justification of the methodology applied for risk evaluation and conclusions for risk mitigation is presented as a model that can be adapted according to the weight of evidence to be submitted in support of the assessment of impact on overall clinical benefit versus risk for the particular situation.

First draft submitted: 1 August 2018; Accepted for publication: 5 December 2018; Published online:

15 February 2019

Keywords: biopharmaceutical • clinical • dossier $\bullet$ immunogenicity $\bullet$ integrated $\bullet$ protein $\bullet$ regulatory $\bullet$ relevance - risk • summary

Although the principles of applying a risk-based approach to the assessment of the undesirable immunogenicity of medicinal products are well established [1-3], effective presentation of the relevant information in regulatory dossiers is challenging because so many pieces of information need to be connected, and regulatory guidance on dossier format has focused on a summary presentation of results of risk evaluation rather than explaining knowledge of product- and patient-related factors that can influence the scale of impact on clinical safety and efficacy. This gap can create important uncertainty about whether pertinent risks have been adequately understood and controlled.

The purpose of this article is to provide practical advice about how to present relevant information in regulatory dossiers, with a particular focus on a model for an Integrated Summary of Immunogenicity (ISI) to be submitted in the Marketing Authorization dossier for a novel medicinal product that has the potential to induce unintended immune responses; the scope includes therapeutic proteins and peptides, cell-based therapies and tissue engineered products.

\section{Investigational studies versus marketing authorization application}

Regulatory authorities encourage sponsors to submit the following information as part of the IND or Investigational Medicinal Product Dossier to support clinical trial applications from the Phase I stage onward: immunogenicity risk assessment, proposed tiered bioanalytical testing strategy and sample timing.

For the marketing authorization application, ICH guidance on the Common Technical Document (CTD) format defines general data requirements, and other regulatory guidelines provide recommendations for points to consider to the immunogenicity assessment. This regulatory guidance is supported by recommendations from learned bodies regarding terminology and data presentation.

\section{ICH guidance on CTD format}

The regulatory assessment of the impact of undesirable immunogenicity on overall clinical benefit and risk is a multidisciplinary exercise that refers to information distributed in different Modules of the Common Technical Document format for Marketing Authorization Applications. 
Table 1. Organisation of immunogenicity-related information in Common Technical Document format.

\begin{tabular}{|c|c|}
\hline CTD module (as per ICH M4E R2 [5]) & Information to include \\
\hline $\begin{array}{l}\text { 2.7.2.4 - Special studies: } \\
\text { Immunogenicity }\end{array}$ & $\begin{array}{l}\text { - Assays used should be briefly described with information about their performance (e.g., sensitivity, specificity, } \\
\text { reliability and validity); the location of detailed information should be cross-referenced } \\
\text { - Incidence, titer, timing of onset and duration of antibody responses for each type of antibody assay used (e.g., IgG } \\
\text { by ELISA, neutralization) } \\
\text { - Relationships of antibody formation to underlying disease, concomitant medication, dose, duration, regimen and } \\
\text { formulation } \\
\text { - Impact of interruptions of therapy on immunogenicity for drugs intended to be given as chronic, continuous therapy } \\
\text { - Analyses of potential clinically relevant correlates of immunogenicity, for example, to determine the extent to } \\
\text { which the presence of antibodies of a particular type or titer appears to correlate with alterations of PK, changes in } \\
\text { PD, loss of efficacy, loss of adverse event profile or development of adverse events. Particular attention should be } \\
\text { paid to events that might be immunologically mediated (e.g., serum sickness) and events that might result from } \\
\text { binding of cross-reactive endogenous substances by antibodies to the administered drug }\end{array}$ \\
\hline $\begin{array}{l}\text { 2.5.3 - Overview of clinical } \\
\text { pharmacology }\end{array}$ & - Interpretation of the results and implications of immunogenicity studies summarized in Module 2.7.2.4 \\
\hline $\begin{array}{l}\text { 5.3.1.4 - Reports of bioanalytical and } \\
\text { analytical methods for human studies }\end{array}$ & - Method validation reports and SOPs for assays used for immunogenicity evaluation across different clinical studies \\
\hline
\end{tabular}

ICH guidelines [4] define the CTD format for Marketing Authorization Applications in EU, USA and Japan; ICH guideline M4E R2 [5] incorporates a very clear recommendation to locate summary information on the clinical immunogenicity assessment in Modules 2.7.2.4 and 2.5.3 of the CTD format, with the associated bioanalytical method validation reports and standard operating procedures (SOPs) located in Module 5.3.1.4 (Table 1).

In addition to the above, individual Clinical Study Reports (CSRs) in Module 5.3 will also contain results from individual studies, and main conclusions about impact of immunogenicity for clinical efficacy and clinical safety are often briefly summarized in Modules 2.7.3 and 2.7.4, respectively.

\section{Limitations of CTD format}

Module 2 of the CTD dossier is intended to provide summary information only, rather than detailed descriptive analyses or discussion. The length of the clinical summary will vary substantially according to the information to be conveyed, but it is anticipated that (excluding attached tables) the clinical summary will usually be in the range of 50-400 pages [5]. Consequently, the general expectation to limit Module 2 to summary information can make it difficult for applicants to present a risk-based justification for the suitability of the immunogenicity evaluation and mitigation strategy that was applied or detailed analyses of clinical results.

The absence of an explicit linkage to a systematic risk assessment process that considers the specific features of the product and the therapeutic population can preclude a full understanding of the nature of the risks and how effectively these have been controlled. Since detectability of treatment-related immune responses and their clinical manifestations can be confounded by methodological factors, it is important for the applicant to explain how the methods used for the risk-based program were designed and validated for suitability.

In addition, because manufacturing and formulation conditions can introduce incremental immunogenicityrelated risks, the omission of information about the product quality control strategy can also weaken strength of conclusions about effectiveness of risk mitigation.

\section{Integrated summary of immunogenicity}

A document format linking the risk analysis to the results of the immunogenicity evaluation has been previously proposed [6], reflecting experience gained from the submission of the ISI in MAA and Biologics License Application (BLA) dossiers. A structured approach was applied for identification of relevant risks, which included product quality- and patient-related factors, and was aligned with recommendations in regulatory guidance documents $[1,2]$. Regulatory assessors from EMA and US FDA found the expanded presentation of data helpful for the immunogenicity review, and both agencies accepted submission of in module 5.3.5.3, on the basis that this enabled more detailed analysis than that presented in module 2.7.2.4.

The revised EMA guidance effective from Dec 2017 [2] endorsed the concept of an immunogenicity summary that integrated the risk analysis with the risk evaluation and mitigation dimensions, as well as indicating that this summary could be submitted either in module 2.7.2.4 or 5.3.5.3 depending on the level of detail. Box 1 identifies the differences introduced by the revised EMA guidance. 
Box 1. EU guidance (EMEA/CHMP/BMWP/14327/2006 Rev 1) compared with ICH M4E R2.

\section{Analysis of risk factors}

- New section to summarize evidence versus uncertainty regarding potential influence of intrinsic and extrinsic factors on risk of clinically impactful immunogenicity

- Alignment of potential risks with strategy for evaluation and mitigation, including linkage to manufacturing, formulation and product quality control strategy

Ideally, an initial Immunogenicity risk assessment would be performed at the Lead Candidate selection stage, and then iteratively updated as new information becomes available during the product development cycle. The output then forms the basis of this section of the ISI

Risk-based immunogenicity program

- Explicit linkage to the output of the immunogenicity risk assessment to justify rationale for design of risk-based program, including frequency and duration of sampling relative to dose regimen and choice of clinical end points for assessing impact on PK, PD, efficacy and safety

- Additional information to help regulatory assessor understand the selection and evolution of bioanalytical methodology and assay controls, validity of assay cut-points, impact of changes on assay performance and linkage to applicable clinical studies in different populations

- Standardization of terminology

Immunogenicity results

- Similar data items as ICH M4E R2, but with option to submit either in module 2.7.2.4 or 5.3.5.3 depending on the level of detail

Submission of the ISI in module 5.3.5.3 enables more extensive presentation of data than is possible in module

2.7.2.4, which can be particularly helpful for discussing impact at individual patient level

Conclusions on the risks of immunogenicity

- Increased emphasis on identification of subpopulations at heightened risk

- Linkage to ongoing risk management

ISI: Integrated Summary of Immunogenicity; PD: Pharmacodynamics; PK: Pharmacokinetics.

\section{Combined use of Modules 2.7.2.4 \& 5.3.5.3 of the CTD format}

While the ISI could be submitted either in Module 2.7.2.4 or 5.3.5.3, an alternative option is to use both Modules, with the full ISI submitted in 5.3.5.3, and a synopsis in 2.7.2.4.

The choice of dossier location for the full ISI will be dictated by the amount of information to present; in the author's experience, the length of the ISI (excluding appendices) can be 80-160 pages of A4 format, which exceeds the size that can be accommodated comfortably into Module 2.7.2.4.

Recent FDA guidance [7] endorses this approach in recommending provision of "brief summaries of the immunogenicity results in relevant places in eCTD section 2.7 Clinical Summary and the full report in section 5.3.5.3."

\section{Model template}

Table 2 presents a model format that seeks to incorporate the headings recommended in the EMA guidance with experience gained from submitting ISI's for a wide range of product types. This model format should be adapted to the particular product scenario, and represents a 'living' document that can be updated to accommodate additional information to support line extensions of the marketing authorization as needed. In the author's opinion, it is not desirable to prescribe a 'globally harmonized' format because there is no ideal that accommodates the diversity of the information to be presented for different product types.

\section{Adapting the starting model for different product types}

The biosimilar situation differs from the innovator product scenario because the risk-based program will be driven primarily by the risk profile established for the reference medicinal product, supplemented by assessment of incremental risk associated with a different manufacturing process. In addition, depending on the bioanalytical strategy applied to monitor the immune response, it may be necessary to present additional evidence (antigenic and antibody equivalence) to support the validity of the methodology to detect potential differences in the immune response to the respective product versions. With respect to results from clinical studies, a detailed comparison of immune response dynamics relative to clinical parameters across the treatment groups will be most critical. 


\section{Table 2. A model template for the Integrated Summary of Immunogenicity}

Executive summary

1. Introduction
Key messaging

- Brief overview of content of extent of data contributing to immunogenicity assessment, including discussion of comparability of drug product to be commercialized relative to material used during clinical development

\section{Analysis of risk factors for PRODUCT $X$}

2.1. Risk assessment: identification of potential risk factors

- Concise discussion of intrinsic and extrinsic risk factors in relation to product attributes, proposed therapeutic use \& existing knowledge

2.1.1. Intrinsic immunogenic potential of molecule

2.1.2. Systems biology

2.1.3. Patient-related factors:

2.1.3.1. Immunologic status and competence of the patient

2.1.3.2. Prior sensitization

2.1.3.3. Route of administration, dose and frequency of administration

2.1.3.4. Genetic status

2.1.3.5. Status of immune tolerance to endogenous proteins

2.1.4. Product quality/linkage to CQA assessment

2.2. Risk assessment: evaluation \& mitigation of risks

2.2.1. Rationale for bioanalytical strategy

2.2.2. Optimizing study design for assessment of clinical impact

2.2.3. ADA results from nonclinical toxicology studies (if relevant)

2.3. Conclusions of immunogenicity risk assessment for PRODUCT $X$

- Explain how the identified risks were addressed

- Justification of sensitivity \& specificity to detect pertinent risks

- Choice of sampling times \& minimization of confounding factors

- Brief summary of relevant observations or justification for nonrelevance

- Tabular summary aligning risk identification to evaluation and mitigation strategy - Justification of effectiveness of manufacturing and product quality control strategy in relation to CQAs that have potential to influence immunogenicity

2.4. Implementation of product-related regulatory advice

- Clarify how agency recommendations were incorporated into the risk-based program

3. Suitability of bioanalytical methods

3.1. Overview of methods

- Tabular summary of methods applied by clinical study with cross-references to supporting documentation

- Schematic diagram of hierarchical test scheme

3.2. ADA assay for screening, confirmatory, specificity and titration

- Clear explanation of each of the following topics to guide the reviewer through the process of demonstrating suitability of the method, including its evolution during the risk-based program

3.2.1. Synopsis of method development

- Design of method in relation to drug and target interference, including evaluation of sample pre-treatment steps and choice of minimum required dilution

\subsubsection{ADA assay format}

- Schematic diagram

3.2.3. Validated performance characteristics for ADA assay

- Tabular summary of results of method validation incorporating amendments

3.2.4. Control of labeled antigen(s)

- Acceptance criteria for controlling lot changes

3.2.5. Negative control matrix

- Source, prescreening prior to pooling and relevance to disease population(s)

3.2.6. Positive control reagents

- Rationale for choice and outline of method of preparation

3.2.7. Justification of competing antigen concentration for confirmatory assay

- Signal inhibition versus competing antigen concentration curve

3.2.8. Specificity controls (if relevant)

- Characterization of domain specificity and cross-reactivity with endogenous factors

3.2.9. Choice of QC concentrations

- Basis for selection and justification of suitability

3.2.10. Selectivity: interference from matrix, drug, target, etc.

- Summarize results to justify adequate selectivity for each of the populations/matrices to be evaluated

3.2.11. Relative sensitivity

- Detectability of surrogate positive control(s) in presence and absence of clinically relevant drug levels

3.2.12. Establishment \& validity of assay cut points

- Explain approaches applied for different clinical studies and justify validity for different populations

3.2.13. System suitability controls

3.3. Neutralizing antibody assay

3.4. PRODUCT $X$ serum/plasma assay

3.4.1. Method description

3.4.2. Influence of ADA on accuracy of PRODUCT $X$ serum assay

- Acceptance criteria applied for validation and clinical sample analysis

- Similar list of subheadings as used for ADA assay

- Impact of ADA on PK assay

- Schematic diagram of assay format

- Evaluation of impact of surrogate ADA positive control on accuracy of assay used to measure drug levels (needed to establish if reported drug concentration and ADA are interdependent or random variables)

\section{Results of clinical evaluation}

4.1. Extent of clinical database

- Tabular summary of number of treated subjects and treatment regimen by study ADA: Anti-drug antibody; AESI: Adverse event of special interest; CQA: Critical quality attribute; ISI: Integrated summary of immunogenicity; PD: Pharmacodynamics; PK: Pharmacokinetics; QC: Quality control. 
Table 2. A model template for the Integrated Summary of Immunogenicity (cont.).

\section{Executive summary}

4.2. Overview of data analysis

4.3. Clinical study $X X X-123$

4.3.1 Study design overview

4.3.2 Drug product batches used

4.3.3 Prior and concomitant medication

4.3.4 Prior history of allergy or drug hypersensitivity

4.3.5 Blinding, collection \& handling of clinical samples for analysis

4.3.6 Clinical sample reconciliation

4.3.7 In study ADA assay performance

4.3.8 ADA / NAb response dynamics

4.3.9 Relationship of ADA/NAb to PK and PD

4.3.10 Relationship of ADA/NAb to efficacy

4.3.11 Relationship of ADA/NAb to safety

4.3.12 Conclusions for study regarding ADA response and clinical impact

4.4. Results from earlier studies

4.X.X

5 Overall conclusions for clinical immunogenicity evaluation and risk mitigation

- Discuss consistency of observations across studies regarding the relationship of the treatment-related immune response to safety and efficacy at the population level and for individual subjects, for example, those developing relatively high ADA titers or antibodies cross-reactive with endogenous counterpart

- Justify adequacy of proposed risk management plan, including product labeling, and describe any ongoing or planned postapproval studies with potential to address uncertainty about impact of immunogenicity on overall benefit versus risk

6 References

APPENDICES:

- Appendix A: definition of AESI

- Appendix B: statistical analysis plan for ISI (if relevant)

- Appendix C: QC charts for assay performance (if not included in bioanalytical reports)

ADA: Anti-drug antibody; AESI: Adverse event of special interest; CQA: Critical quality attribute; ISI: Integrated summary of immunogenicity; PD: Pharmacodynamics; PK: Pharmacokinetics; QC: Quality control.

In the case of a multi-domain therapeutic protein, there may be very different risk profiles associated with individual domains. Thorough evaluation of the specificity of signals detected in pre- and post-treatment samples may be necessary. The same consideration applies to PEGylated therapeutic proteins. For some novel constructs, unexpected cross-reactivity with pre-existing antibodies has been observed, resulting in a need to identify structural motifs in the product that bind to endogenous factors.

Use of nonmammalian host cells for protein expression may be associated with product-related variants and process-derived impurities that represent potential factors for incremental immunogenicity risk. These factors should be considered within the risk analysis section and, if warranted, evaluated as part of the risk-based program. This may necessitate additional testing of the specificity of immune responses, in terms of both pre-existing and treatment-emergent antibodies.
- Explain how the results were analyzed, including definition of terminology and data outputs

- Schematic of sampling time-points relative to treatment period(s) and - Tabular summary

- Brief comment on control of potential confounding influence on immune

- Indicate whether subjects with history of nonserious allergy or drug

- Describe sample collection and handling for bioanalysis, including operator blinding

explanation of reasons for missing samples

- Tabular summary of false ADA positive results for pre- and post-treatment samples ind reliability of assay cut-point)

- Tabular and graphical summaries of ADA/NAb positive frequency versus time - Descriptive analyses to indicate influence of ADA/NAb positive status, and of ADA titer quartile, on Ctrough; discuss whether ADA could interfere with accuracy of influence

- Tabular summary to indicate incidence of AESI by ADA/NAb positive status severity of AESI

- Critical discussion of weight of evidence versus uncertainty, taking into account

- Similar subheadings as for main study, with each study presented in order of decreasing weight of evidence 
Box 2. Hierarchy of concerns.

Potential consequence (in order of decreasing severity)

- Induction of anti-drug antibodies that cross-react with endogenous counterparts

- Allergic-type hypersensitivity/anaphylaxis

- Complement activation-related pseudo-allergy

- Immune complex-related hypersensitivity

- Reduced pharmacodynamic response/efficacy

- Altered pharmacokinetics

- Compromise of subsequent treatment with related products

- Uncertain long-term clinical impact

\section{Table 3. Categorization of factors that could interact to influence the probability and severity of undesirable} consequences of immunogenicity.

\begin{tabular}{|ll|}
\hline Category & Properties \\
\hline Intrinsic factors & - Structural and functional properties of product that could contribute to intrinsic immunogenic potential \\
\hline Systems biology & - Abundance and uniqueness/redundancy of function of endogenous counterparts of the drug product \\
& - Location and function of target \\
\hline Conditions of use & - Clinical dosing regimen, including route of administration, level and frequency of dosing \\
\hline Patient related & - Characteristics of the target population, including immune competence, prior exposure to the drug product or to related \\
& products and genetic factors that may influence immune recognition/responsiveness \\
\hline Product quality & - Manufacturing process and rigor of product quality control \\
& - Extent of analytical methods \\
& - Formulation and drug product stability \\
& - Comparability of clinical versus commercial product \\
\hline This table is based on data from $[1,2]$. &
\end{tabular}

\section{Purpose of the risk analysis}

The risk analysis serves two main objectives, namely to:

- Aid understanding of the potential causes of clinically impactful immunogenicity for the particular product and intended therapeutic use;

- Justify adequacy of the risk evaluation and mitigation activities to support a suitably informed decision on benefit versus risk in the target population.

These objectives reflect the recommendation in FDA guidance [1] that:

"If clinically relevant immune responses are observed, sponsors are encouraged to study the underlying mechanism and identify any critical contributing factors. These investigations can facilitate development and adoption of potential control and mitigation strategies, including modification of product formulation and screening of higher risk patients."

This requires a thoughtful analysis of the different factors that could interact to influence the probability and severity of clinical manifestations, allied to active minimization of variables that might confound detection and interpretation of signals.

\section{Risk-based approach}

The established framework for the risk analysis is to consider the potential clinical manifestations of immunogenicity for the particular scenario (product + intended use), and then to define activities for evaluation and criteria for risk mitigation for each potential consequence. The consequences can be listed according to severity Box 2, corresponding to the "hierarchy of concerns" that was articulated by Amy Rosenberg in 2003 [8] .

A question-based approach [6,9] can be useful to triage the relevance of individual intrinsic and extrinsic risk factors (Table 3) for the product and its intended use, encompassing the full range of points to consider as recommended in regulatory guidance $[1,2]$. This then provides a basis for definition of specific risk evaluation and mitigation activities that are aligned to the relevance of the individual risk factors for the particular scenario.

The potential influence of confounding variables for signal detection and interpretation (Box 3) can also be discussed in relation to clinical study design and justification of analytical and bioanalytical strategy. 
Box 3. Potentially confounding factors for risk evaluation

Control via clinical study design

- Genotypic \& phenotypic variability of target population

- Influence of pre-existing, cross-reactive antibodies

- Influence of comorbidities and concomitant medications

Control by method selection, validation and testing

- Levels of product-related variants and process-derived impurities

- Performance of bioanalytical methods

\begin{tabular}{|c|c|c|}
\hline Potential consequence & Risk evaluation & Risk mitigation \\
\hline $\begin{array}{l}\text { Allergic-type } \\
\text { hypersensitivity/anaphylaxis }\end{array}$ & $\begin{array}{l}\text { Preclinical: } \\
\text { - Comparative ex-vivo basophil activation testing (healthy } \\
\text { humans vs. atopic subjects) } \\
\text { Clinical: } \\
\text { - Monitoring of timing and severity of clinical symptoms of } \\
\text { infusion-related reactions relative to pre-existing and } \\
\text { treatment-emergent ADA with cross-reactivity to } \\
\text { non-human glycans (additional specificity tier incorporated } \\
\text { in ADA testing scheme) } \\
\text { - Measurement of serum tryptase } \\
\text { - Follow-up investigation of IgE ADA \& ex-vivo basophil } \\
\text { activation test in subjects with potential immune-mediated } \\
\text { AE's in Phase III study }\end{array}$ & $\begin{array}{l}\text { 1. Molecular design to minimize nonhuman glycans } \\
\text { associated with expressed protein } \\
\text { 2. Absence of ex-vivo basophil activation in naive or treated } \\
\text { subjects } \\
\text { 3. Negligible serum tryptase in treated patients } \\
\text { 4. No subjects fulfilling NIAID FAAN criteria for anaphylaxis } \\
\text { 5. No severe systemic hypersensitivity reactions reported in } \\
\text { clinical program } \\
\text { 6. AE's not related to drug-specific IgE }\end{array}$ \\
\hline
\end{tabular}

The output of the risk analysis can be presented in a tabular summary that explicitly links the risk evaluation and mitigation activities to each identified potential consequence. Table 4 illustrates how this information might be presented to address a potential risk associated with the capacity of a nonmammalian host cell line to introduce potentially allergenic glycans as a post-translational modification of a therapeutic protein.

This format can then be extended to incorporate each of the clinical consequences that were identified as being potential relevant for the intended therapeutic use of the product. It can be helpful to present this type of tabular summary format as part of the IMPD or IND dossier to support clinical trial applications from the Phase I stage onward, because this provides a clear and transparent synopsis of the information needed by the regulatory assessor. The same format, updated with additional information as relevant, can also be used as the summary of the risk analysis for the ISI.

\section{Role of in silico \& in vitro data on intrinsic immunogenic potential}

Although not a mandatory requirement, it can be relevant to summarize in silico and in vitro data to explain the relative intrinsic potential of an investigational product when compared with molecules that have an established clinical immunogenicity profile. For example, if the primary amino acid sequence of investigational product incorporates promiscuous high affinity MHC Class II binding motifs, and has an endogenous counterpart with a nonredundant physiological function, this could translate into a need for more rigorous control of extrinsic factors.

It is also possible that expression of a therapeutic protein in E.coli could increase intrinsic immunogenic risk via the addition of an $\mathrm{N}$-terminal methionine residue if this represents a new anchor residue for MHC Class II binding. Thus, understanding impact of $\mathrm{N}$-terminal or other amino acid modifications associated with the expression system can be instructive for immunogenicity risk mitigation.

\section{Scope of product quality-related information to include in ISI}

ICH guidance on pharmaceutical development [10] encourages the identification of critical quality attributes to justify suitability of manufacturing and product quality control. Typically, the potential contribution of individual product attributes to the scale of immunogenicity is one of the risk dimensions that is taken into account in this exercise. Accordingly, inclusion in the Risk Analysis chapter of the ISI of the output of the CQA assessment identifying product quality attributes having highest potential to influence immunogenicity can be helpful. This 


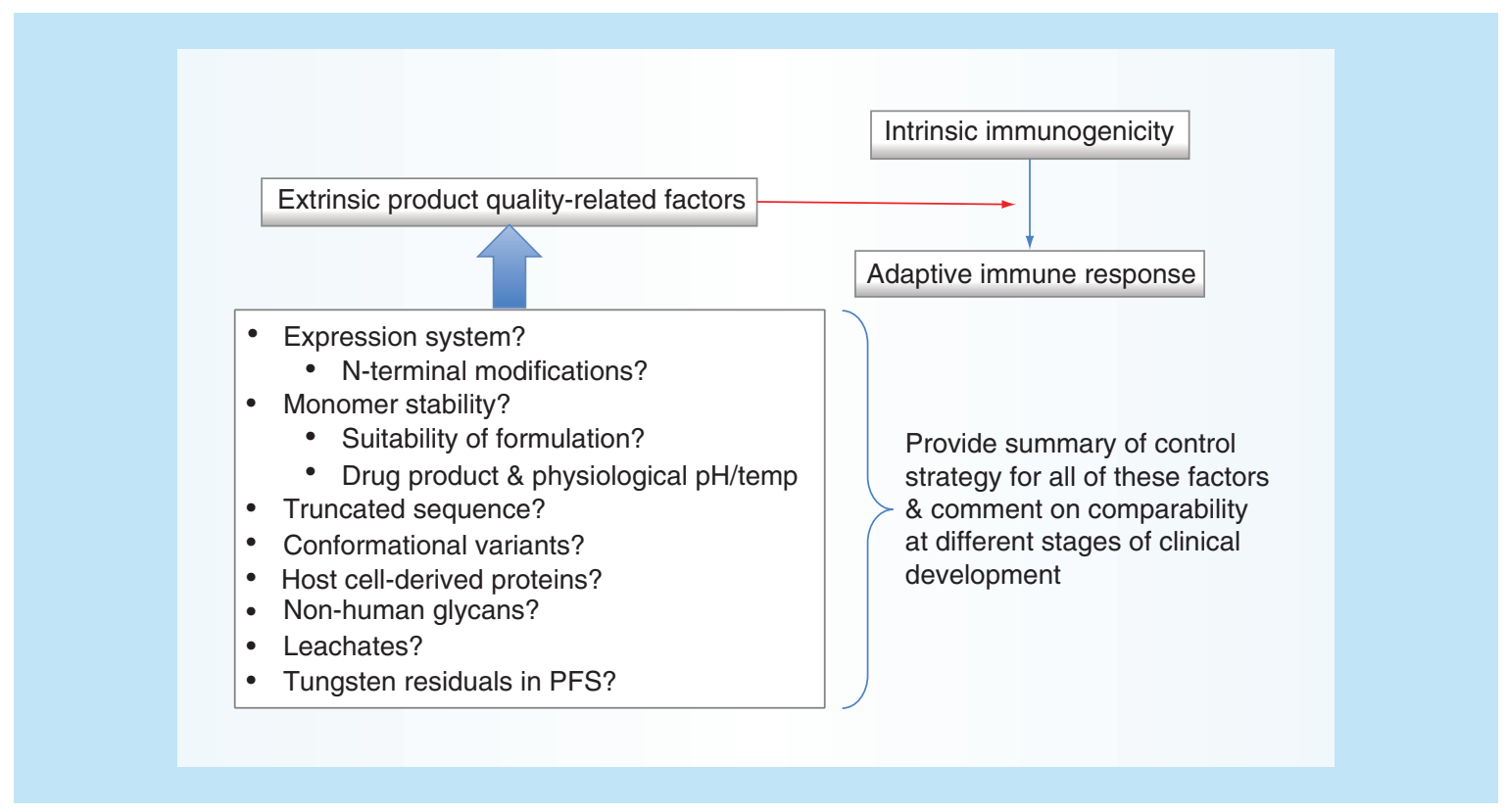

Figure 1. Product quality-related factors to be considered in risk assessment. PFS: Pre-filled syringe.

can be supplemented by information to indicate control of levels of high molecular weight variants, aggregates, subvisible particles, host cell-derived impurities, nonhuman glycans and other factors as relevant, with crossreferences to Module 3 for the source data. Information on stability of the active substance in the drug product formulation-primary container combination to be commercialized will also be relevant, and comparability to the material evaluated in clinical studies should be discussed. Figure 1 illustrates the product quality-related variables that might be referred to in the risk analysis.

\section{Linkage to method validation reports \& SOPs submitted in Module 5.3.1.4}

For many marketing authorization applications, the regulatory assessor is obliged to work in a fragmented manner to review method validation reports for multiple iterations of an anti-drug antibody (ADA), and/or NAb and/or drug concentration $(\mathrm{PK})$ assay; often, these reports do not clearly explain the relationship between the different method versions described and which clinical studies these apply to; to make the situation more difficult, there may be multiple amendments to these reports. It is not unusual to find more than 25 documents for review in Module 5.3.1.4.

The ISI chapter justifying the suitability of bioanalytical methods provides an opportunity to help the regulator navigate through the rationale for choice of method and critical reagents, evolution of conditions and relationship to different clinical studies. In addition to providing an overview of the methodology and validated performance characteristics, including a brief summary of important data from the method development phase (e.g., establishment of minimum required dilution), the validity of the assay cut-points for different clinical populations could be discussed.

It is worth emphasizing that $80 \%$ of questions arising during the MAA review process refer to bioanalytical aspects: the aim of the bioanalytical chapter in the ISI is to reduce this avoidable burden for the benefit of both the reviewer and the applicant.

\section{Relationship of ISI to clinical study reports \& other CTD modules}

The CSRs submitted in Module 5.3 should contain a summary of results of the descriptive analyses of the $\mathrm{ADA}$ and NAb response in each treatment group, as defined in the Statistical Analysis Plan (SAP). Typically, the Bioanalytical Report containing the supporting data is included as an Appendix to the CSR. The ISI then provides the opportunity to present more detailed analyses of the data to illustrate the relationships of the ADA and NAb signals to PK, PD, efficacy and safety parameters for each clinical study. Conclusions about clinical impact within 


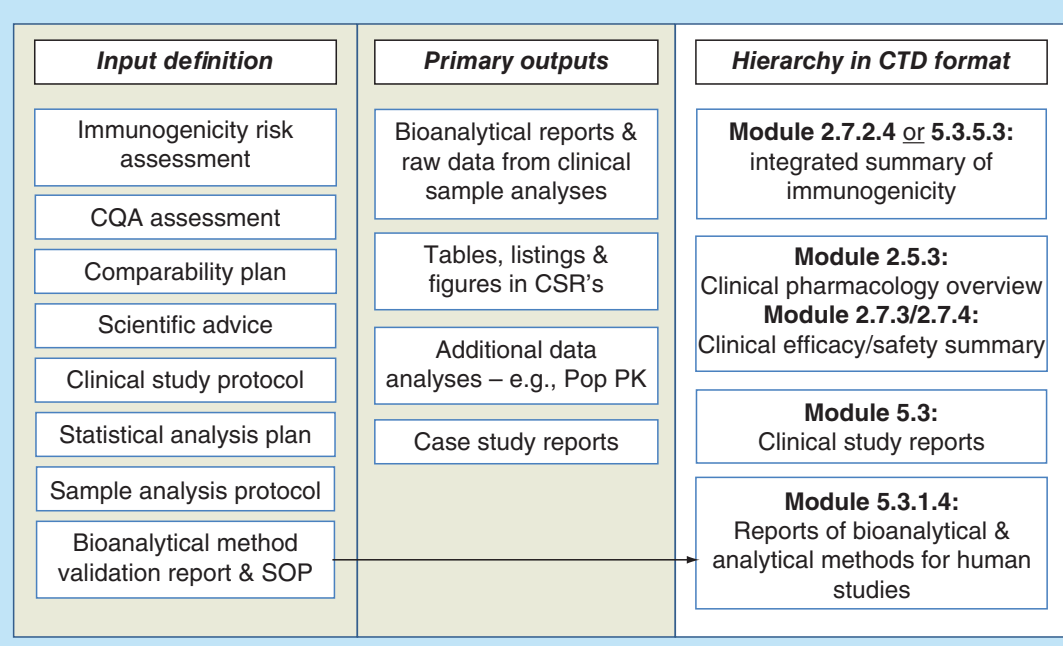

Figure 2. Relationship of data elements to Common Technical Document format.

CQA: Critical quality attribute; CSR: Clinical study reports; PK: Pharmacokinetics; SOP: Standard operating procedure.

and across individual clinical studies may then be fed into the relevant Module 2 summaries. Figure 2 illustrates the relationship of the input and primary output elements to the organization of the CTD format.

\section{Timing of ISI preparation}

Effectively, the preparation of the ISI commences with the risk assessment performed at the lead candidate selection stage, which is then iteratively updated during clinical development. The risk assessment forms the major part of the first chapter of the ISI, linking the identified risks to the control measures that are applied at the time of the marketing authorization application. A mock version of the complete ISI can then be compiled some 12-18 months ahead of the dossier submission, with placeholders for the results of ongoing clinical studies. As soon as the tables, listing and figures from the final clinical study are available, the remaining data elements can be incorporated in the ISI.

\section{Definition of ISI data inputs}

Definition of descriptive analyses of the bioanalytical and clinical data inputs for the ISI represents a critical preliminary step in the process. Helpful guidance is available that explains different options for data presentation [11,12] that can be used for defining the specific analyses to be performed. If not explicitly defined in the main SAP for each relevant clinical study, additional descriptive analyses could be detailed in a supplementary document, sometimes referred to as the "ISI SAP". This then serves to assist the data management team to anticipate any additional tables, listing and figures required for the ISI, without prejudice to the analyses already planned in the main SAP. The primary data elements that have been most useful in the author's experience for presentation in the ISI are:

- Relative sensitivity of ADA assay in presence of residual drug;

- Multidomain product: reactivity versus different domains;

- $\mathrm{ADA} / \mathrm{NAb}$ frequency at each time-point;

- Pre-existing ADA prevalence versus treatment-emergent/boosted ADA incidence;

- Distribution of ADA titer at each time-point;

- $\mathrm{ADA}$ titer quartile versus $\mathrm{C}_{\text {trough }}$ (box-and-whisker plot);

- ADA titer quartile versus primary efficacy end point (box-and-whisker plot);

- Spaghetti plot of $\mathrm{C}_{\text {trough }}$ or $\mathrm{PD} /$ efficacy versus time for $\mathrm{ADA}$ positive $\mho \mathrm{ADA}$ negative subpopulations;

- Individual patient profiles of $\mathrm{ADA}$ titer versus $\mathrm{C}_{\text {trough }}$ versus $\mathrm{PD} / \mathrm{efficacy}$;

- Receiver operating characteristic based analysis of clinically meaningful titer for diminished efficacy [13];

- Timing and severity of adverse events of special interest relative to ADA status and ADA titer. 
ADA assay data granularity

\begin{tabular}{|l|}
\hline \multicolumn{1}{|c|}{ Item } \\
\hline Patient ID/code \\
Assay run no. \\
\hline Assay run date \\
\hline Age \\
\hline Gender \\
\hline Treatment group \\
\hline Sampling time point \\
\hline Corresponding low QC value in screening assay (RLU) \\
\hline Corresponding high QC value in screening assay (RLU) \\
\hline Corresponding negative control value in screening assay (RLU) \\
\hline Plate-specific screening assay cut-point \\
\hline Screening assay result (RLU) \\
\hline Screening assay assignment: positive/negative \\
\hline \\
\hline Low QC value in confirmatory assay (RLU) \\
\hline High QC value in confirmatory assay (RLU) \\
\hline Negative control value in confirmatory assay (RLU) \\
\hline Confirmatory assay result (RLU) \\
\hline Percent inhibition in the confirmatory assay \\
\hline Confirmatory assay assignment: positive/negative \\
\hline Titer of confirmed positive samples \\
\hline Concentration of on-board drug at time of sampling \\
\hline
\end{tabular}

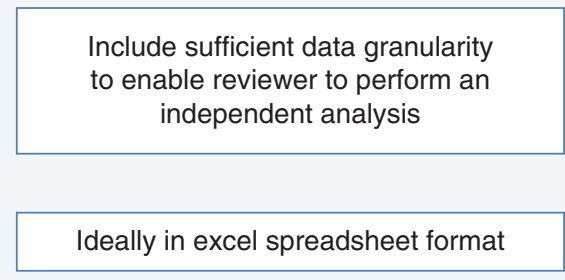

Appendix to:

- CSR or

- Sample analysis report or

- ISI

Plan into data transfer agreement with CRO and assign treatment ID following data lock \& un-blinding

Figure 3. ADA assay data granularity.

ADA: Anti-drug antibody; CRO: Contract research organization; CSR: Clinical study reports; ID: Identity; ISI: Integrated Summary of Immunogenicity; QC: Quality control; RLU: Relative light unit.

It is important to emphasize that while population $\mathrm{PK}$ analyses exploring the relationship of ADA positive/negative status can be helpful, their value may be seriously compromised if - as is often the case [14] - the accuracy of the drug concentration measured by a ligand-binding assay is influenced by ADA interfering with binding of the drug to its target ligand (or to an anti-idiotypic antibody reagent). For this reason, it is essential to validate the drug concentration (PK) assay for ADA interference, and to exclude data for subjects who have an ADA titer above the threshold for interference in the PK assay.

Granularity of raw data from clinical sample analyses using ADA, NAb and PK assays may also need to be considered to ensure that Bioanalytical reports are adequately detailed to facilitate independent analysis by regulatory assessors; if necessary, data transfer agreements can define supplementary outputs, for example, an Excel spreadsheet incorporating the data fields shown in Figure 3, which corresponds with requests frequently received from regulatory agencies during the review of the marketing authorization application dossier.

Discussion of the timing and severity of safety signals in relation to the ADA/NAb response can be facilitated by individual subject profiles, supplemented with results of relevant laboratory parameters (e.g., complement activation products, serum tryptase and drug-specific $\operatorname{IgE}$ ). Adverse events should be described using standard terminology, in other words, MedDRA preferred terms and System Organ Classes, and recommended definitions of anaphylaxis [14]. The ISI could also present graphical profiles for subjects with relatively high ADA titers to illustrate the time-course of $\mathrm{ADA} / \mathrm{NAb}$ signals relative to drug concentration, $\mathrm{PD}$ and efficacy parameters - which can be very helpful for assessing the scale of impact, if any, of clinically relevant immunogenicity for the 'worst-case' subpopulation.

\section{Linkage to risk management plan}

The closing part of the ISI should address the questions:

- What is the overall risk for the target population, in balancing probability versus consequences of an immune response to the product?

- How will this risk be managed? 
The regulatory assessor needs to understand the benefit-risk balance for individual subjects as well as that at the population level. Thus, discussion of clinical impact in subjects with upper quartile ADA titer could be important, as well as longer term outcomes in the ADA/NAb-positive subpopulation.

In the case where clinically impactful immunogenicity was observed, it would be relevant to justify the adequacy of the current database to support approval in association with proposed monitoring and cautionary measures to be applied in clinical practice. The value of additional post-authorization evaluation, for example, from a prospectivelydefined observational cohort study or a patient registry, might also be discussed. Consideration should also be given to risks associated with off-label use, and for patients to be treated with related products. In extreme cases, it may be necessary to define conditions for modification or withdrawal of treatment.

\section{Future perspective}

More effective presentation of immunogenicity-related information in regulatory submissions will help regulators to make a fully informed and balanced assessment of the clinical impact, if any, of immunogenicity of biopharmaceutical products. In the case of uncertainty, appropriate risk management activities may be aligned with the scale of potential risks for the proposed therapeutic use of the product.

\section{Executive summary}

- Although the risk-based approach for management of potential immunogenicity of therapeutic proteins and peptides is well established, the extent to which different intrinsic and extrinsic factors have been identified and evaluated to achieve effective risk mitigation is often not clearly communicated in regulatory dossiers.

- Dispersion of the relevant information across different modules of the Common Technical Document (CTD) format makes it difficult for the applicant to integrate multi-disciplinary elements into a complete analysis. In addition to dispersed location, the level of granularity of data presentation is restricted by the desire to present only summary information in Module 2 of the CTD format.

- Inclusion of an Integrated Summary of Immunogenicity (ISI) in Module 5 of the CTD format can facilitate linkage of the risk assessment to the results of the immunogenicity evaluation in a manner that facilitates the regulatory review.

- This article provides a model ISI format to indicate the scope of information that could be included, bearing in mind that the actual format and content should be adapted to reflect the nature of the product and the population to be treated. This model is consistent with the principles recommended in regulatory guidance.

- Consideration of the product quality control strategy relative to the intrinsic immunogenic properties of the molecule, in addition to variables associated with the patient population and conditions of use of the product, provides a basis for the risk assessment that informs the design of the evaluation and justifies the adequacy of the level of risk mitigation for the intended therapeutic use.

- Suitability of bioanalytical methodology for the intended clinical application can be explained to help the regulatory reviewer navigate the individual method validation reports and associated documents to be submitted in Module 5.3.1.4. This should include an explanation of how methods have evolved during clinical development, as well the rationale for choice and control of critical reagents, and for the frequency and duration of monitoring.

- Presentation of detailed descriptive analyses of the bioanalytical and clinical data inputs in the ISI provide granularity to enable conclusions about clinical impact of the detected signals.

- Planning the data inputs for the ISI well ahead of the time helps to ensure availability of the requisite information. The article discusses the utility of different data inputs.

- The concluding part of the ISI then provides an opportunity for the applicant to propose an ongoing risk management strategy to address uncertainties at the time of marketing authorization.

\section{Open access}

This work is licensed under the Attribution-NonCommercial-NoDerivatives 4.0 Unported License. To view a copy of this license, visit http://creativecommons.org/licenses/by-nc-nd/4.0/

\section{Acknowledgements}

This article reflects experience gained through interactions with many regulators and industry colleagues since 1998 . The concept of the Integrated Summary of Immunogenicity was originally introduced as an element of the Biologics License Application (BLA) for vaccine products, and then adapted to address undesirable immunogenicity of therapeutic proteins. The model format for the ISI presented in this article would not have been possible without the vision and support of G Dallmann, P Kurki and J Pedrasvasconcelos. 
Financial \& competing interests disclosure

$P$ Chamberlain is a member of the NDA Advisory Board and provides consulting services to the pharmaceutical industry; he is also Principal of bioLOGICA Consulting SARL (France). The author has no other relevant affiliations or financial involvement with any organization or entity with a financial interest in or financial conflict with the subject matter or materials discussed in the manuscript apart from those disclosed.

No writing assistance was utilized in the production of this manuscript.

\section{References}

1. Guidance for industry: immunogenicity assessment for therapeutic protein products, FDA, August 2014 (2014).

www.fda.gov/ucm/groups/fdagov-public/@fdagov-drugs-gen/documents/document/ucm338856.pdf

2. Guideline on Immunogenicity assessment of therapeutic proteins (EMEA/CHMP/BMWP/14327/2006 Rev 1). Committee for Medicinal Products for Human Use (CHMP) (2017).

www.ema.europa.eu/docs/en_GB/document_library/Scientific_guideline/2017/06/WC500228861.pdf

3. Koren E, Smith HW, Shores E et al. Recommendations on risk-based strategies for detection and characterization of antibodies against biotechnology products. J. Immunol. Methods 333(1-2), 1-9 (2008).

4. ICH guidelines. www.ich.org/products/guidelines

5. ICH M4E R2 Common technical document for the registration of pharmaceuticals for human use - efficacy, Step 5, 15 July 2016 EMA/CPMP/ICH/2887/1999 (2016).

www.ema.europa.eu/en/ich-m4e-common-technical-document-registration-pharmaceuticals-human-use-efficacy

6. Chamberlain P. Addressing immunogenicity-related risks in an integrated manner. Regulatory Affairs Pharma, Jan 2011, 10-15 (2011). www.ndareg.com/wp-content/uploads/2014/04/RAP_January_2011_Paul-Chamberlain.pdf

7. Guidance for industry: immunogenicity testing of therapeutic protein products - developing and validating assays for anti-drug antibody detection, FDA January

2019 (2019). www.fda.gov/ucm/groups/fdagov-public/@fdagov-drugs-gen/documents/document/ucm629728.pdf

8. Rosenberg AS. Immunogenicity of biological therapeutics: a hierarchy of concerns. Dev. Biol. (Basel) 112, 15-21 (2003).

9. Chamberlain P. Presenting an immunogenicity risk assessment to regulatory agencies. In: Immunogenicity of Biopharmaceuticals. Chapter 13, van de Weert M, Horn MØller E (Eds). Springer, NY, USA, 239-258 (2008).

10. ICH guideline Q8 (R2) on pharmaceutical development, Step 5, EMA/CHMP/ICH/167068/2004, 22 June 2017 (2017). www.ema.europa.eu/docs/en_GB/document_library/Scientific_guideline/2009/09/WC500002872.pdf

11. Shankar G, Arkin S, Cocea L et al. American Association of Pharmaceutical Scientists. Assessment and reporting of the clinical immunogenicity of therapeutic proteins and peptides-harmonized terminology and tactical recommendations. AAPS J. 16(4), 658-673 (2014).

12. Rup B, Pallardy M, Sikkema D et al. Standardizing terms, definitions and concepts for describing and interpreting unwanted immunogenicity of biopharmaceuticals: recommendations of the Innovative Medicines Initiative ABIRISK consortium. Clin. Exp. Immunol. 181(3), 385-400 (2015).

13. Steenholdt C, Bendtzen K, Brynskov J, Thomsen OØ, Ainsworth MA. Cut-off levels and diagnostic accuracy of infliximab trough levels and anti-infliximab antibodies in Crohn's disease. Scand. J. Gastroenterol. 46(3), 310-318 (2011).

14. Sailstad JM, Amaravadi L, Clements-Egan A et al. Consensus and recommendations of a global harmonization team on assessing the impact of immunogenicity on pharmacokinetic measurements. AAPS J. 16(3), 488-498 (2014).

15. Manivannan V, Decker WW, Stead LG, Li JT, Campbell RL. Visual representation of National Institute of Allergy and Infectious Disease and Food Allergy and Anaphylaxis Network criteria for anaphylaxis. Int. J. Emerg. Med. 2(1), 3-5 (2009). 